\title{
Compressible Flow Simulations with an Spectral Difference Method and Using High-Order Boundary Treatment
}

\section{T. Martini ${ }^{1}$}

Aerodynamics Division, Departamento de Ciência e Tecnologia Aeroespacial, DCTA/IAE/ALA, São José dos Campos, SP

A. R. B. Aguiar ${ }^{2}$

Student, Graduate Program in Space Sciences and Technology, Departamento de Ciência e Tecnologia Aeroespacial, DCTA/ITA

J. L. F. Azevedo ${ }^{3}$

Aerodynamics Division, Departamento de Ciência e Tecnologia Aeroespacial, DCTA/IAE/ALA

\begin{abstract}
The present work addresses several aspects associated with an efficient use of the high-order Spectral Difference (SD) method for the simulation of compressible flows. Flows of interest are assumed to be adequately modeled by the two-dimensional (2-D) Euler or the 2-D Navier-Stokes equations. Issues associated with the use of curved boundaries are discussed. Namely, for special cases where highly stretched meshes are required, the problem of auto-intersecting elements is highlighted. Further developments on general order meshes with an attention to node positioning are also discussed. Motivated primarily by the needs of compressible viscous flows, the use of radial basis functions and mesh optimization will be discussed in order to accommodate the interior nodes. Furthermore, an important validation test for curved meshes is addressed, in order to demonstrate the capability implemented.
\end{abstract}

Key-words. High-Order Meshes, Compressible Flow, Radial Basis Functions, Mesh Optimization.

\section{Introduction}

High-order numerical schemes represent the natural extension of current computational fluid dynamics (CFD) methods, which were developed over the past thirty years for aerospace simulations. The current generation methods are mostly 2nd-order accurate and have achieved a level of maturity and robustness desirable for everyday deployment in aeronautical engineering scenarios. Likewise, several complementary methods were developed for time integration, convergence acceleration, shock capturing and for dealing with geometric complexities. However, there are many problems that cannot be fully simulated using low-order methods, such as vortex dominated flows. Moreover, high-order methods

\footnotetext{
${ }^{1}$ tiaramartini@gmail.com

${ }^{2}$ ufabc.andre@gmail.com

3 joaoluiz.azevedo@gmail.com
} 
offer the possibility to reduce simulation costs for given solution accuracy levels, when compared to low-order schemes.

There is room for improvement in many areas for high-order methods that must be pursued before they can compete with industrial-level CFD solvers. Computational resource requirements and run time are typical metrics used to classify a specific method or a combination of methods in a CFD solver. High-order schemes must cope with implicit schemes, limiters or filters and mesh manipulation techniques that also need to be superior, in comparison with the low-order counterparts. Therefore, a high-order method coupled with a low-order mesh with linear elements, for instance, will degrade the accuracy of the method near the domain boundaries. In order to overcome this problem and to fully realize the advantages of higher-order methods, a correct description of curved boundaries is mandatory. The solver is currently enabled with implicit methods for convergence acceleration whereas the mesh capabilities are still being recently developed.

The main contribution of the present work is to extend high-order curved mesh capabilities so as to fully explore high-order methods potential with respect to aerospace applications. A study assessing the effects of a second order curved mesh generated from a B-spline reconstruction revealed an improvement in the results for inviscid flow over two-dimensional configurations [1].

The major motivation is the development of the ability of handling complex geometries within both inviscid and viscous flows over aerospace configurations. This leads to two major concerns with respect to both applications which are the extensions of the previous studies. The first one is to be able to provide a general order curved mesh representation and specify point locations by making sure there is no instability problems [2]. Lastly, while auto-intersecting elements, or inverted elements, may not be a problem for the inviscid case, viscous simulations bring the need to obtain a reasonable resolution for the boundary layer. The refinement near the wall requires the propagation of the deformation near into the interior domain in such a way that a highly refined quadrilateral mesh is still capable of being curved. To deal with this issues two different approaches are discussed: Radial Basis Functions and Mesh Optimization.

\section{High-Order Boundary Treatment}

In order to render the high-order reconstruction process manageable, it is of great importance to consider curved meshes which better represent more complex geometries. This also has a side effect of reducing the required number of cells in the domain. Furthermore, the use of piecewise linear approximations of a curved boundary leads to imprecise solutions when using high-order schemes [3]. Therefore, it is mandatory to use a precise description of the curved geometry of the boundary so as to obtain meaningful and accurate results. The point is that performing a correct boundary treatment ought to lead to more efficient results than refining the grid on its own.

The approach used to generate the high-order mesh in the present paper consists of four subsequent steps, which can be summarized as creating the boundary representation of the model, generating a linear coarse mesh upon it, defining new nodes for each domain 
cell and, ultimately, projecting these new nodes onto the model geometry boundaries. This approach is considered an a-posteriori procedure as suggested in Ref. [4] and it overcomes some of the obstacles of directly generating a high-order mesh whilst taking advantage of the robustness of current linear mesh generators [5].

Although interior faces might not be deformed for inviscid problems, the ongoing development is to propagate the curvature to the interior domain using Radial Basis Functions or Mesh Optimization, which will be discussed later. In this sense, new interior nodes will be placed on the linear edges using a correct prescribed movement dependent on the curvature of the geometry. This is necessary to handle highly stretched meshes that may be needed to capture boundary layer properties on a viscous flow.

A commonly used geometric entity that describes geometries in 2-D is the Non-Rational B-Spline (NURBS) curve. The procedures undertaken in this paper assume that geometries are generated through this type of B-spline and exported using the IGES (Initial Graphics Exchange Specification) [7] standard file exchange format. The IGES file format provides the necessary information to reconstruct the B-splines [6] that recovers the geometry. The Rational B-spline curve definition is represented by Equation (1) as follows:

$$
B(u)=\frac{\sum_{i=0}^{n} w_{i} P_{i} N_{i, k}}{\sum_{i=0}^{n} w_{i} N_{i, k}},
$$

where $u$ is the parameter value of the B-spline, that varies in an interval of 0 to $100 \%$ of the length of the curve. The fact that B-splines are defined as a function of its length parameter will be crucial on the node projection step. The weights $w_{i}$ are associated with each control point, defined by $P_{i}$. An arbitrary amount of $n+1$ control points are used to describe the informations of a B-spline of degree $k$. The basis functions, $N_{i, k}$, used in the construction of the B-spline, use the information of a knot vector $T_{i}$ to be recursively defined by the Cox-de Boor formula [6]:

$$
\begin{gathered}
N_{i, 0}(u)= \begin{cases}1, & \text { if } u_{i} \leq u<u_{i+1} \\
0, & \text { elsewhere }\end{cases} \\
N_{i, k}(u)=\left(\frac{u-T_{i}}{T_{i+k}-T_{i}}\right) N_{i, k-1}(u)+\left(\frac{T_{i+k+1}-u}{T_{i+k+1}-T_{i+1}}\right) N_{i+1, k-1}(u),
\end{gathered}
$$

Often, the concern of viscous flows is the analysis of properties inside a thin boundary layer. The dimension of a boundary layer is excessively small when compared to the dimension of the model and, more importantly, huge gradients are observed within it, which suggests that the mesh near this region ought to be well refined in order to capture acceptable results. Moreover, the higher the Reynolds number, the more prohibitive will be the mesh capable of capturing reasonable results. The higher-order meshes, in this sense, may bring the advantage of properly representing the boundary, thus, reducing the number of required elements to obtain the same results. It is crucial to understand that these aspects have to be considered so as to generate a reliable and robust high-order mesh.

Still within the context of a solution inside a boundary layer, a problem concerning the transformation of a linear to a curved mesh known as "element inversion" needs to 
be highlighted. This problem comes from the fact that the properties inside a boundary layer mostly vary on the direction orthogonal to the solid boundary, which allows the mesh to be relatively less refined in the direction tangent to the boundary. In short, cells that are capable of capturing properties inside the boundary layer have, in general, a high aspect ratio which, in turn, makes those cells flat. In the case of overly flattened cells, it is possible for the geometry to cross several cells at once and, in an attempt to curve only the boundary cells, it will intersect with its own edge [8]. This element inversion is translated to a negative area - or volume, in 3-D -, which directly affects the calculation of the jacobians and, consequently, spoils the solution. Figure 1 depicts a case of a highly stretched quadrilateral cell fitted to a highly curved boundary which leads to an invalid element.

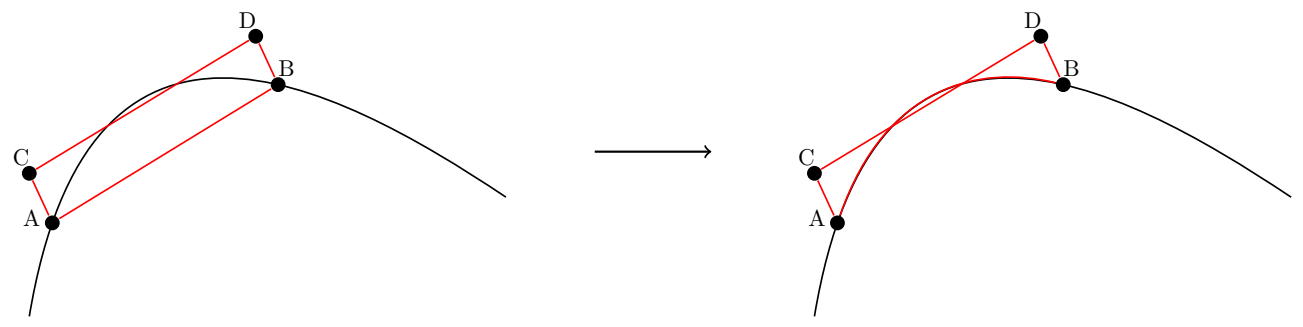

Figure 1: Mapping of a non-valid curved element.

\section{Radial Basis Function Formulation}

For typical viscous aerospace problems, the gradients observed near the wall requires the mesh to be highly stretched normal to the configuration. The process of curving the boundaries described in Sec 2, in this case, generally generates problems of cells crossing each other. The problem is that the curvature is being applied exclusively to the boundary mesh nodes. The idea of using Radial Basis Functions (RBF) is to propagate this curvature into the interior domain.

$\mathrm{RBF}$ are used to provide node movement for all mesh nodes given a prescribed movement of some boundary nodes. The displacement of all mesh nodes without prescribed movement is obtained by a sum of basis functions that depends on the Euclidean distance between nodes that have prescribed movement and the interior nodes of the mesh. The new displaced mesh is obtained in such a way that its topology is preserved.

\section{Mesh Optimization}

A different approach to deal with tangled meshes, a mesh with inverted elements, consist of using mesh optimization. The optimization approach has shown promised results $[9,13-15]$. 
Therefore a cost function $f_{c}$ is defined in order to evaluate the "quality" of the elements. The cost function depends on a quality measure capable to identify inverted elements and penalize this with a high cost. The idea is untangle the mesh and, at the same time, improve the quality of all elements. Typical quality measures involve the condition number and determinant of the Jacobian matrix $[10,12]$, mean-ratio $[9,13]$, among others $[11,14,15]$. This procedure defines an optimization problem:

$$
\text { Minimize } f_{c} \text {, s.to } v \in \Omega
$$

where $v$ is a vector with nodes coordinates and $\Omega$ represents the constraints that define the problem geometry.

Practical methods to solve (4) are iterative. So, given an initial approximation $v^{0}$ a sequence $v^{1}, v^{2}, \ldots$ is generated, in order to approximate it, at each new iteration, to a solution of the problem. Among the iterative process, the gradient method, or some variation, is the most used by the aerospace community. We compare this methods with a very common one among the optimization community, the Newton method.

After the optimization process, the new interior nodes coordinates are optimized, according to function $f_{c}$.

\section{Results}

The numerical results presented here attempt to verify resolution and robustness of the high-order SD method coupled with the different techniques listed in the paper. For the results discussion, density is made dimensionless with respect to the freestream condition and pressure is made dimensionless with respect to the freestream density times the speed of sound squared.

An important validation for the curved meshes is the case of a subsonic inviscid flow over a circular cylinder. A flow with freestream Mach number of 0.2 is simulated and the solution for the Euler equations in this domain is expected to be twice symmetrical, given that compressibility effects are small for this freestream Mach number. This test demonstrates that spurious results can be observed for high curvature geometries, even though a continued sequential refinement is performed over the linear mesh. The correct representation of the geometry using higher order meshes, however, handles this effect properly. A quadrilateral linear mesh composed by 16 cells in both radial and azimuthal directions $(16 \times 16)$ is defined. Afterwards, even finer meshes with $32 \times 32$ and $64 \times 64$ cells are considered to assess the effects of using 2nd-order meshes.

The following results are obtained with a 3rd-order method, in which the solution is reconstructed by a 2nd-order polynomial (P2). In this sense, the order of the polynomial that reconstructs the mesh is compatible with the one that reconstructs the solution. The results in Fig. 2 (a) show that a linear mesh exposes the flow to kinks in the geometry in such a way that the flow can separate, even though an inviscid formulation is used for the present computations. Hence, the order of the polynomial used to reconstruct the solution is higher than the one used to reconstruct the geometry and, furthermore, the geometry is linear. Therefore, lower-order erroneous solutions build-up from the coarse geometry 
representation. When a curved, valid quadratic mesh representation is considered, the results are clearly symmetric and the numerical oscillations are effectively handled, i.e., they are eliminated, see Fig. 2 (b)-(c).

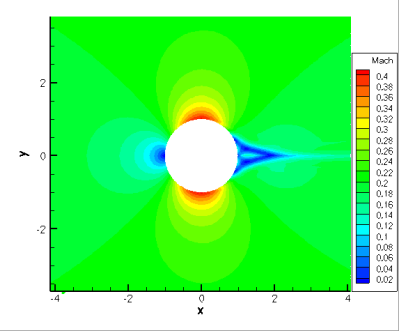

(a)

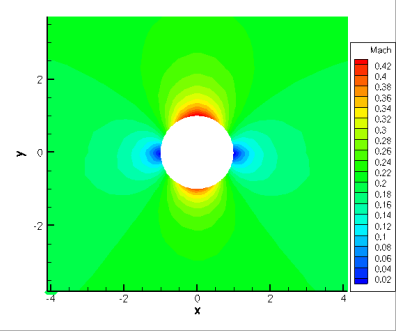

(b)

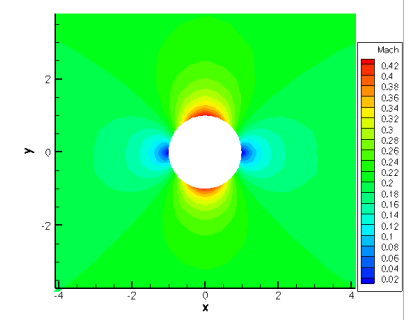

(c)

Figure 2: Mach contours for a $16 \times 16$ mesh using a 3rd-order SD method considering: (a) linear mesh; (b) optimized quadratic mesh; (c) RBF quadratic mesh.

\section{Concluding Remarks}

The paper discusses several issues and results for an implementation of the high-order SD method for compressible flows. The SD method uses a simple universal reconstruction to yield high-order polynomial approximations of the solution. The main contribution of the present work is to extend the previous framework that handles simple inviscid meshes to a more appropriate approach of curvature propagation so as to obtain a smooth high-order mesh that properly conforms even in the case of a highly stretched mesh.

\section{Acknowledgements}

The authors gratefully acknowledge the support for the present research provided by Conselho Nacional de Desenvolvimento Científico e Tecnológico, CNPq, under the Research Grants No. 309985/2013-7, No. 400844/2014-1, No. 443839/2014-0 and No. 150329/2016-4. The work is also supported by Fundação de Amparo à Pesquisa do Estado de São Paulo, FAPESP, under Research Grant No. 2013/07375-0. The support provided by Fundação Coordenação de Aperfeiçoamento de Pessoal de Nível Superior, CAPES, through a graduate scholarship for the second author, is also greatly appreciated.

\section{References}

[1] F. M. Moreira, E. J. C. Breviglieri, and J. L. F. Azevedo. Implicit Spectral Difference Method Solutions of Compressible Flows Considering High-Order Meshes. 22nd AIAA Computational Fluid Dynamics Conference, 2015-3195, 2015. 
[2] J. S. Hesthaven. From electrostatics to almost optimal nodal sets for polynomial interpolation in a simplex. SIAM Journal on Numerical Analysis, 35:655-676, 1998.

[3] F. Bassi, and S. Rebay. High-Order Accurate Discontinuous Finite Element Solution of the 2D Euler Equations. Journal of Computational Physics, 138(2):251-285, 1997.

[4] A. G. Peiró, X. Roca, J. Peraire, and J. Sarrate. High-Order Generation on CAD Geometries. Proceedings of the VI International Conference on Adaptive Modeling and Simulation, 2013.

[5] Z. J. Wang, K. Fidkowski, R. Abgrall, F. Bassi, D. Caraeni, A. Cary, H. Deconinck, R. Hartmann, K. Hillewaert, H. T. Huynh, N. Kroll, G. May, P. Persson, B. van Leer, and M. Visbal. High-Order CFD Methods: Current Status and Perspective. International Journal for Numerical Methods in Fluids, 72(8):811-845, 2013.

[6] C. de Boor. A Practical Guide to Splines, Springer-Verlag, New York, 2001.

[7] W. B. Gruttke. The Initial Graphics Exchange Specification (IGES) v. 6.0, IGES/PDES Organization, 1995.

[8] P. Persson, and J. Peraire. Curved Mesh Generation and Mesh Refinement Using Lagrangian Solid Mechanics. 47th AIAA Aerospace Sciences Meeting and Exhibit, 2009 .

[9] L. Freitag, P. Knupp, T. Munson, and S. Shontz. A comparison of optimization software for mesh shape-quality improvement problems. Technical report, Argonne National Lab., IL (US), 2002.

[10] L. A. Freitag and P. M. Knupp. Tetrahedral mesh improvement via optimization of the element condition number. International Journal for Numerical Methods in Engineering, 53(6):1377-1391, 2002.

[11] H. Hoppe, T. DeRose, T. Duchamp, J. McDonald, and W. Stuetzle. Mesh optimization. In Proceedings of the 20th annual conference on Computer graphics and interactive techniques, 19-26, 1993.

[12] P. Knupp. Matrix norms and the condition number. In Proceedings of 8th International Meshing Roundtable, 13-22, 1999.

[13] J. Kim, M. Shin, and W. Kang. A derivative-free mesh optimization algorithm for mesh quality improvement and untangling. Mathematical Problems in Engineering, 2015 .

[14] S. L. Karman. Adaptive optimization-based smoothing for tetrahedral meshes, In 53rd AIAA Aerospace Science Meeting, 2015.

[15] S. L. Karman, J. T. Erwin, R. S. Glasby, and D. Stefanski. High-order mesh curving using wcn mesh optimization, In 46th AIAA Fluid Dynamics Conference, page 3178, 2016. 